\title{
Commissioning and Performance of the ATLAS Inner Detector with proton-proton Collisions at the LHC
}

\author{
Antonio Limosani*, on behalf of the ATLAS Collaboration \\ University of Melbourne, Australia \\ E-mail: antoniol@unimelb.edu.au
}

\begin{abstract}
The ATLAS Inner Detector is a composite tracking system consisting of silicon pixels, silicon strips and straw tubes in a $2 \mathrm{~T}$ magnetic field. Its installation was completed in August 2008 and the detector has taken part in data taking with colliding LHC beams. Utilising collision data we present studies of the individual sub-systems, which include measurements of the Lorentz angle, timing, noise characteristics and cluster reconstruction efficiency. We also report results of the post-collisions alignment of the detector geometry and probe the agreement between data and simulation in the early stages of track reconstruction at ATLAS.
\end{abstract}

35th International Conference of High Energy Physics

July 22-28, 2010

Paris, France

${ }^{*}$ Speaker. 


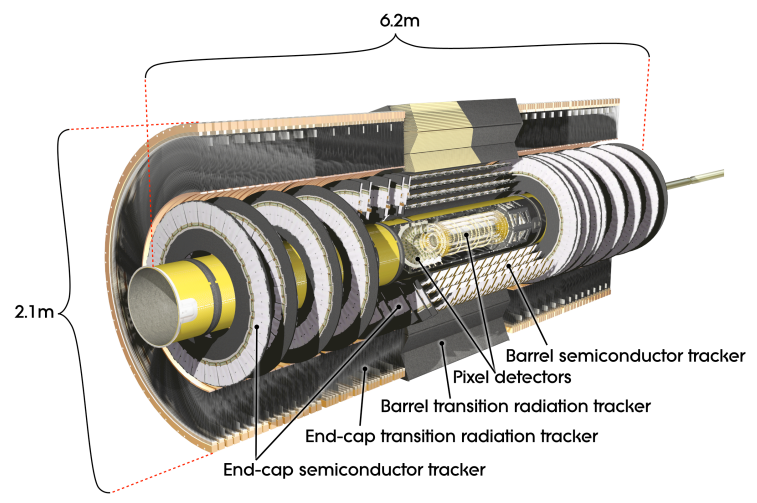

(a)

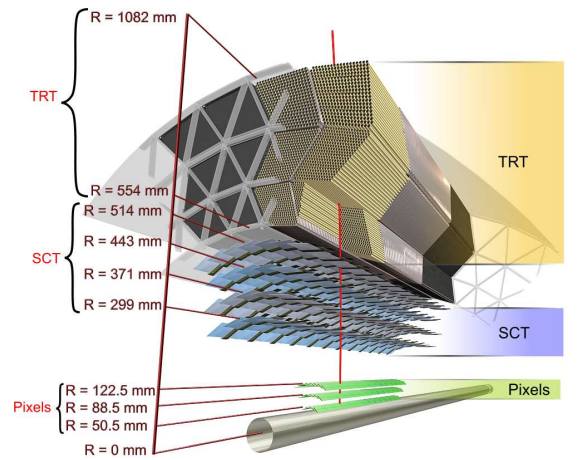

(b)

Figure 1: (a) Layout of the ATLAS Inner Detector. (b) Zoom in of a barrel section of the Inner Detector.

\section{The ATLAS Inner Detector}

The ATLAS Inner Detector (ID) [1] measures the trajectories of charged particles. It is immersed in a solenoidal field of $2 \mathrm{~T}$ to enable measurements of particle momenta. Extending outward from the interaction point it consists of three sub-detector systems: the Pixel Detector, Semiconductor Tracker (SCT), and Transition Radiation Tracker (TRT). The layout of the Inner Detector and a cross-sectional view are displayed in Fig. 1a and Fig. 1b, respectively. It occupies a cylindrical volume of diameter $2.1 \mathrm{~m}$ and length $6.2 \mathrm{~m}$, and for particles emanating from beam collisions, provides a solid angle coverage of $2 \pi$ and \pm 2.5 in azimuth and pseudorapidity respectively.

Pixel Detector modules are arranged into three barrel layers and three discs in each endcap. A barrel module consists of high-granularity silicon pixel detectors. Altogether 1744 modules provide 80 million readout channels. High-granularity ensures low occupancy, which is necessary for robust track pattern recognition. The resolution on measurements of track impact parameters and vertex finding is dominated by the precision of the pixel detectors. The intrinsic barrel spatial resolution is $10 \mu \mathrm{m}$ and $115 \mu \mathrm{m}$ in $r \phi$ and $z$ respectively.

The SCT consists of 4088 modules of silicon strip detectors arranged into four barrel layers and nine discs in each endcap. Each module is made up of back to back silicon wafers offset by 40 mrad to provide a two-dimensional space-point measurement. Barrel wafers have a strip pitch of $80 \mu \mathrm{m}$ resulting in an intrinsic resolution of $23 \mu \mathrm{m}$. Endcap modules are trapezoidal in shape with strips fanning out from and perpendicular to the beam line. Endcap strip pitches thus vary from 50 $\mu \mathrm{m}$ to $90 \mu \mathrm{m}$. Altogether there are 6.3 million strips. The spatial resolutions for barrel modules in $r \phi$ and $r z$ are $17 \mu \mathrm{m}$ and $500 \mu \mathrm{m}$ respectively.

The TRT consists of 298,000 $4 \mathrm{~mm}$ diameter straw-tube detectors. The straws are aligned along and fanned perpendicular to the beamline in the barrel and endcap sections respectively. The inside of a tube is layered with a thin coat of aliminium foil, which acts as a cathode. A $30 \mu \mathrm{m}$ diameter gold-plated anode wire is placed at the tube centre. Tubes are filled with a Xe- $\mathrm{CO}_{2}-\mathrm{O}_{2}$ gas mixture that ionises when traversed by a charged particle. The TRT provides about $30 r \phi$ measurements per track yielding a spatial resolution of approximately $170 \mu \mathrm{m}$. The TRT helps to distinguish tracks of electrons from hadrons through detection of transition radiation. The radiation 


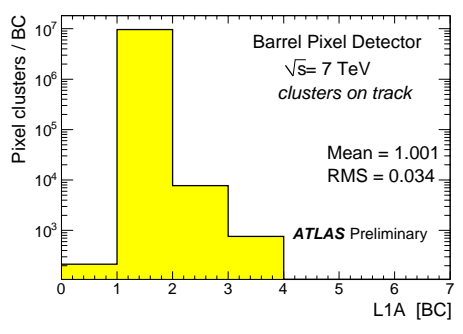

a)

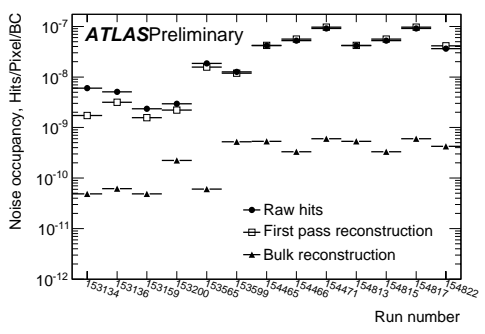

b)

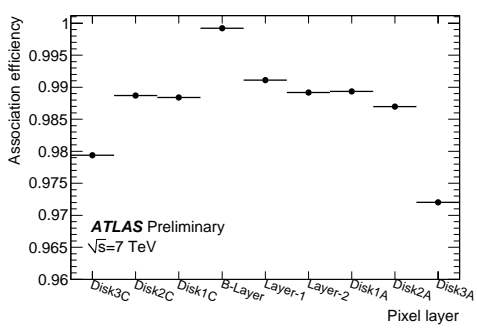

c)

Figure 2: a) Timing of pixel cluster read-out. b) Pixel Detector occupancy in randomly triggered events with empty bunches. c) Efficiency for a track to have a hit associated when crossing a Pixel Detector layer.

is generated by polypropylene foils and fibres placed between straws.

\section{Sub-detector Status and Performance}

The Inner Detector was fully operational when first proton collisions were provided by the LHC in late November 2009. Currently only 44 pixel modules are inactive, which contributes to the total inactive fraction of $2.64 \%$. The Lorentz angle was measured to be $211.3 \pm 2.6 \mathrm{mrad}$ in $7 \mathrm{TeV}$ collision data and is well modeled in the simulation. The readout of a pixel hit is well timed into the bunch crossing (BC) as shown in Fig. 2a. The noise occupancy, shown in Fig 2b, has been measured in empty bunches, and after masking of noisy channels, is shown to be less than $10^{-9}$ well below the $10^{-7}$ requirement. This translates to less than 0.2 noise hits per event when reading out 5 BCs. The hit efficiency (the probability to register a hit given an incident particle) is above 98\% for all but one endcap disk, as shown in Fig. 2c, where dead modules are excluded from the calculation.

Only 30 out of 4088 SCT modules to date are inactive. Altogether including less serious faults, there is a total inactive fraction of $0.97 \%$. The mean time-bin for the SCT hit readout has a mean close to 1, demonstrating the SCT is well timed in to BCs. The noise occupancy, as shown in Fig 3a, is in good agreement with simulation except at low numbers of noisy strips, where noise in the simulation is appropriate for the read-out of one BC, whilst in data they are read out in 3 BCs. The hit efficiency for the barrel layers, as plotted in Fig. $3 b$, is $99.8 \pm 0.1 \%$, and is the same in the endcaps, which is above the design goal of $99 \%$. The Lorentz angle is well measured in each of the layers and in agreement with model expectations.

Currently $98 \%$ of channels in the TRT are functioning. Straws measure the drift time (t) for the signal to reach the low threshold, which is converted to a track-to-wire distance (R), where the Rt relation is shown in Fig. 4a. The TRT hit reconstruction efficiency is about $94 \%$ for data and $95 \%$ for simulation, and is plotted in Fig. $4 \mathrm{~b}$. The number of high-threshold hits is sensitive to transition-radiation. Electrons produce more transition-radiation than pions as shown in Fig. 4c. The electrons are specially selected from photon conversion candidates. Discrimination is available in the range from $1 \mathrm{GeV}$ to around $250 \mathrm{GeV}$. 


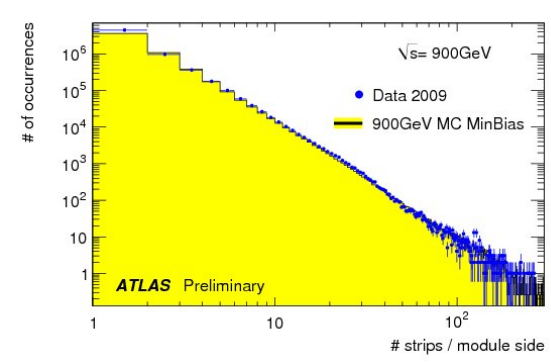

a)

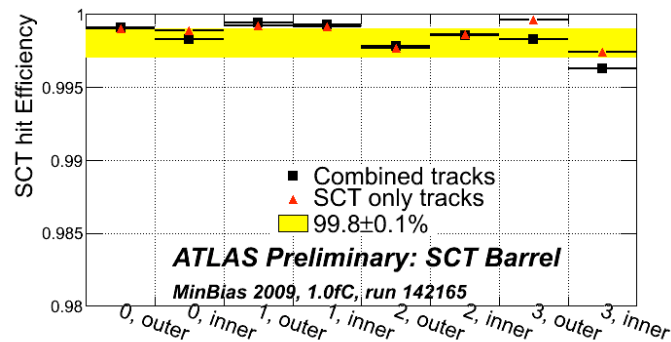

b)

Figure 3: a) The frequency of the number of SCT noisy strips per module side. b) Intrinsic module efficiency for tracks measured in the SCT Barrel.

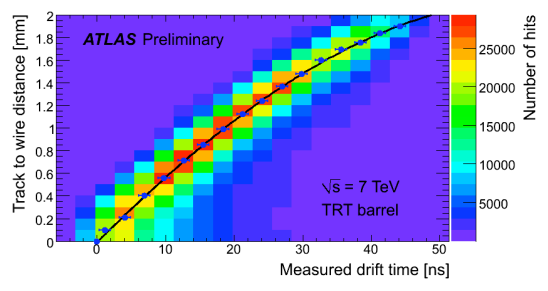

a)

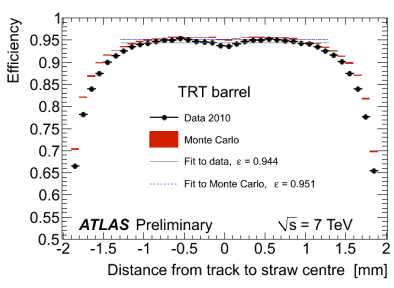

b)

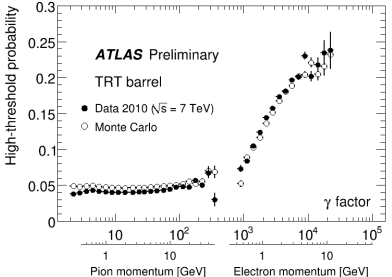

c)

Figure 4: a) TRT Rt relation. b) TRT hit reconstruction efficiency. c) TRT transition-radiation onset.

\section{Alignment Status}

Alignment of the Inner Detector geometry is implemented using a track-based approach, where alignment and track parameters are derived from the minimisation of a global chi-square, which consists of track-to-hit distances known as residuals. Prior to collisions, aligmnent constants were derived from cosmic-ray data. A comparison of residuals derived from post- and pre-collisions alignment constants for the SCT barrel and endcap are plotted in Fig. 5a and Fig. 5b respectively. The SCT endcap, which received relatively less illumination from cosmic rays, benefits most from post-collisions alignment. The Pixel and TRT alignment constants are also improved though the effect is less pronounced. The deviation of the mean transverse track impact parameter from zero is reduced under the post-collisions regime, as shown in Fig. 5c. The residuals have proved to be stable over a timescale of months, demonstrating that there have been no significant changes in the module positions and orientations during data taking. These results [2] show the performance is approaching that of a perfect alignment as incorporated in the simulation.

\section{Tracking Performance}

Very good agreement of tracking performance between data and MC was demonstrated through the measurement of charged particle multiplicities using the first collision data [3]. Latest results at very low momentum [4], in the range from $100<p_{T} / \mathrm{MeV}<500$, show excellent agreement between data and simulation, as shown in Fig. 6a. Excellent agreement has now also been demonstrated in the pattern recognition stage of track reconstruction [5], at the level where track seeds are 


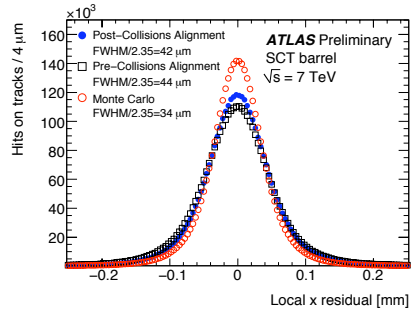

a)

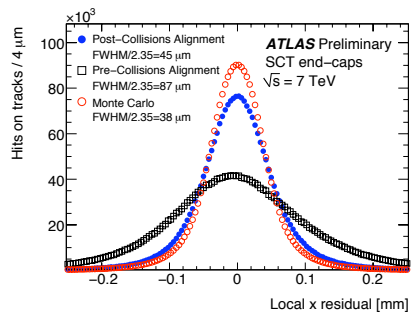

b)

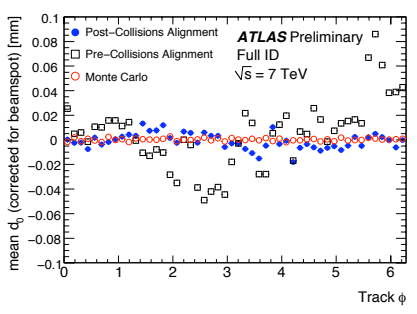

c)

Figure 5: The residuals for tracks in the SCT a) barrel and b) endcap. c) The mean transverse impact parameter of tracks corrected for the beam spot.

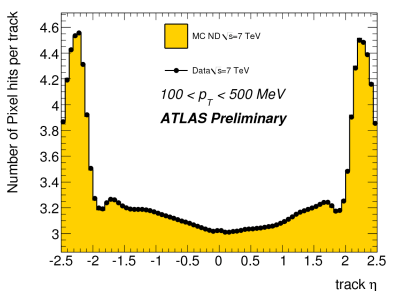

a)

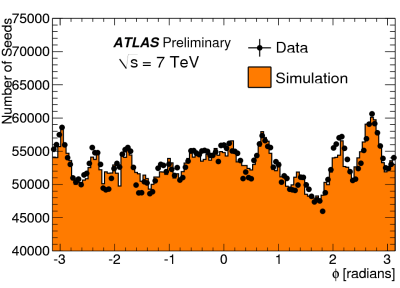

b)

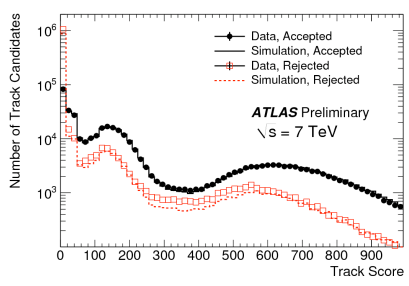

c)

Figure 6: a) Number of pixel hits per track. b) Azimuthal angle distribution of track seeds and c) track candidates as a function of track score in the track pattern recognition stage.

formed from 3 space-points and resultant track candidates are resolved into the final track collection via the use of a track score, as shown in Fig. $6 \mathrm{~b}$ and Fig. $6 \mathrm{c}$ respectively.

\section{Conclusion}

The ATLAS Inner Detector is working very well, with an operational performance exceeding expectations. Alignment of the ID geometry in data is approaching that of the ideal. The tracking has been studied in fine detail down to the level of pattern recognition, where data and simulation have shown to be in very good agreement. More detail on performance as measured with collision data can be found elsewhere [6].

\section{References}

[1] G. Aad et al. [ATLAS Collaboration], [arXiv:0901.0512] [hep-ex].

[2] ATLAS Collaboration, Alignment Performance of the ATLAS Inner Detector Tracking System in 7 TeV proton-proton collisions at the LHC, ATLAS-CONF-2010-067.

[3] G. Aad et al. [ATLAS Collaboration], Phys. Lett. B 688 (2010) 21 [arXiv:1003.3124 [hep-ex]].

[4] ATLAS Collaboration, Charged particle multiplicities in pp interactions for track $p_{T}>100 \mathrm{MeV}$ at $\sqrt{s}=0.9$ and $7 \mathrm{TeV}$ measured with the ATLAS detector at the LHC, ATLAS-CONF-2010-046.

[5] ATLAS Collaboration, Performance of the ATLAS Silicon Pattern Recognition Algorithm in Data and Simulation at $\sqrt{s}=7 \mathrm{TeV}$, ATLAS-CONF-2010-072.

[6] G. Aad et al. [ATLAS Collaboration], JHEP 1009 (2010) 056 [arXiv:1005.5254 [hep-ex]]. 